\title{
Reconstruction of Orbital Fracture with the Aid of Mirror Imaged 3D Printed Models
}

\author{
Samy Saeed El Naas Farag Allah*, Mahmoud Ahmad Abdallah, Wael Abd Almagid Almohandis \\ Department of Oral and Maxillofacial Surgery, Faculty of Dental Medicine, Al-Azhar University, Cairo, Egypt \\ * Corresponding author: Samy Saeed El Naas Farag Allah, Mobile: (+20)1069007128, \\ email: samy.saeed.edu@gmail.com
}

\begin{abstract}
Background: The use of mirror-imaging techniques with the aid of three-dimensional computed tomographic (3DCT) scanning and 3D printing for reconstruction of orbital fractures could improve the outcome and maximize the functional and esthetic reconstruction of traumatized orbit.

Objective: The aim of the present study was to evaluate reconstruction of orbital fracture with the aid of mirror imaged 3D printed models. Patients and Methods: A total of 10 patients with unilateral orbital fractures were selected from those having maxillofacial injuries at the emergency room of Sayed Galal University Hospital, AlAzhar University, Cairo. Patients were divided randomly into two groups (five patients each); Group I: patients were treated with mirror imaging technique and fabrication of a rapid prototype skull model. Group II: patients were treated through the traditional technique without fabrication of a rapid prototype skull model.

Results: At one, three and six months postoperatively, all patients affected with diplopia within both groups have a total recovery. The difference was statistically significant. The traumatized orbits were reduced and fixed in both groups through either technique and there was a statistically insignificant difference in mean orbital volume in unaffected side and the affected side (immediately postoperatively).

Conclusion: It could be concluded that the use of mirror image rapid prototype skull model for treatment of orbital fractures has no advantage over the traditional method.
\end{abstract}

Keywords: orbital fracture, diplopia, orbital volume, rapid prototyping

\section{INTRODUCTION}

Orbital floor injuries, alone or in combination with other facial fractures, are one of the most encountered midfacial fractures. Having an incidence of $10-25 \%$ from the total facial fractures and the most common age group is the third decade of life. Significant complications can occur as a result of these injuries, including enophthalmos, persistent diplopia, vertical dystopia, restriction of gaze and even blindness ${ }^{(1-4)}$.

The majority of cases of orbital fractures require reconstruction of the orbital floor to support the globe position and restore the shape of the orbit. The most important factor in minimizing postoperative complications related to globe position is restoration of the orbital bones to their correct anatomic position. The unique and complex anatomy of the orbit requires significant contouring of the implants to restore the proper anatomy ${ }^{(5,6)}$.

Technological advancements in imaging and surgical computer planning may assist the surgeon in this task ${ }^{(7-10)}$. One of the most promising emerging technologies is the ability to use computer planning software to create a mirror image overlay (MIO) on a craniofacial computed tomographic CT scan. This involves duplicating the contralateral, non- traumatized, facial region and superimposing its skeleton onto the fractured, displaced region ${ }^{(11,12)}$.

The use of mirror-imaging techniques with the aid of three-dimensional computed tomographic (3DCT) scanning and 3D printing for reconstruction of orbital fractures could improve the outcome and maximize the functional and esthetic reconstruction of traumatized orbit ${ }^{(\mathbf{1 3}, \mathbf{1 4})}$.

Changes of the orbital volume may affect the final treatment outcomes. Precise alignment of the broken orbital bones indicates proper reconstruction. This study has examined the volumetric changes of orbit associated with orbital fractures before and after reconstruction with the aid of mirror imaged rapid prototype skull model.

The aim of the present study was to evaluate reconstruction of orbital fracture with the aid of mirror imaged 3D printed models.

\section{PATIENTS AND METHODS}

This study included a total of 10 patients with unilateral orbital fractures who were selected from those having maxillofacial injuries, attending at emergency room of Sayed Galal University Hospital, Al-Azhar University, Cairo. All patients were informed about the scope of the study and signed an informed written consent form. 


\section{Ethical approval:} obtained.

Approval of the ethical committee was

Patients were divided randomly into two groups (five patients each); Group I: patients were treated with mirror imaging technique and fabrication of a rapid prototype skull model. Group II: patients were treated through the traditional technique without fabrication of a rapid prototype skull model.

\section{Preoperative evaluation and preparation: \\ $>$ Clinical examination:}

Clinical examination, including general, local and ophthalmological examinations, was done. An ophthalmologist was consulted to assess the visual acuity, diplopia (using Hess chart test to be recorded as either normal, diplopia at divergent angle gaze, or diplopia at primary gaze position) and perform forced duction test (FDT) if the gaze was found to be restricted. Patients were evaluated clinically for the following: skin wounds, periorbital hematoma, and infraorbital nerve affection. (Figure 1).

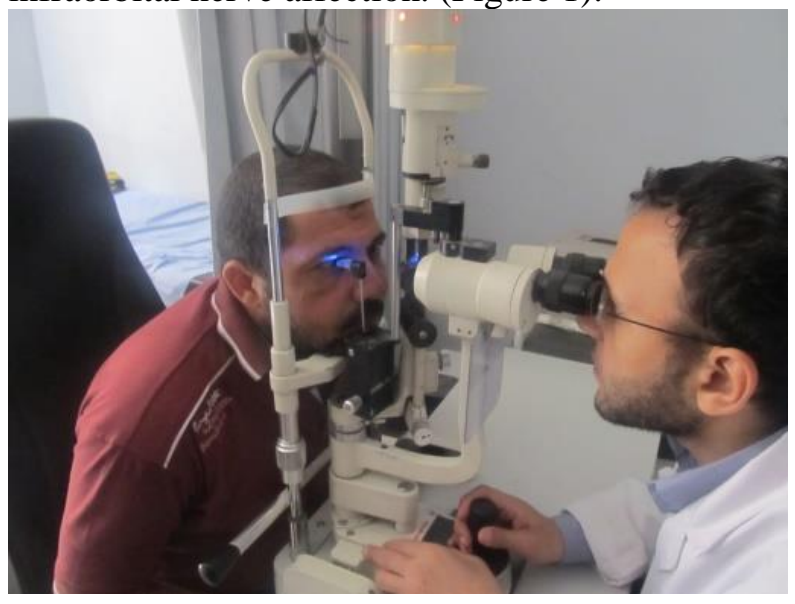

Figure (1): Ophthalmologic examination of the patient.

\section{Radiographic examination:}

CT scanning of the skull was performed to assess the following:

- Location, direction and complications of the fracture.

- Measurement of the volume of both of the two orbital cavities (the traumatized and the contralateral).

- Design and manufacture a rapid prototype skull model of the mirror imaged unaffected orbit.

\section{Preparation of STL model:}

With the aid of the 3DCT scan of the skull, rapid prototype skull model was manufactured. Scanned DICOM (Digital Imaging and Communications in Medicine) images were imported to Mimics software (Materialize, Leuven, Belgium). The normal uninjured side of the orbital ZMC region was reflected onto the contralateral injured side.

The mirror imaged STL model data were used to manufacture a rapid prototype skull model by 3D printing technique (Dent 1 DLP 3D printer using FunToDo). This life-size rapid prototype skull model which resembles the individual skull model of the uninjured side was used to precontour the titanium mesh and / or miniplate (s) which were used in the reconstruction of the orbit and / or ZMC. The precontoured plates / mesh were sterilized carefully so as not to distort the predetermined contouring. (Figure 2) 


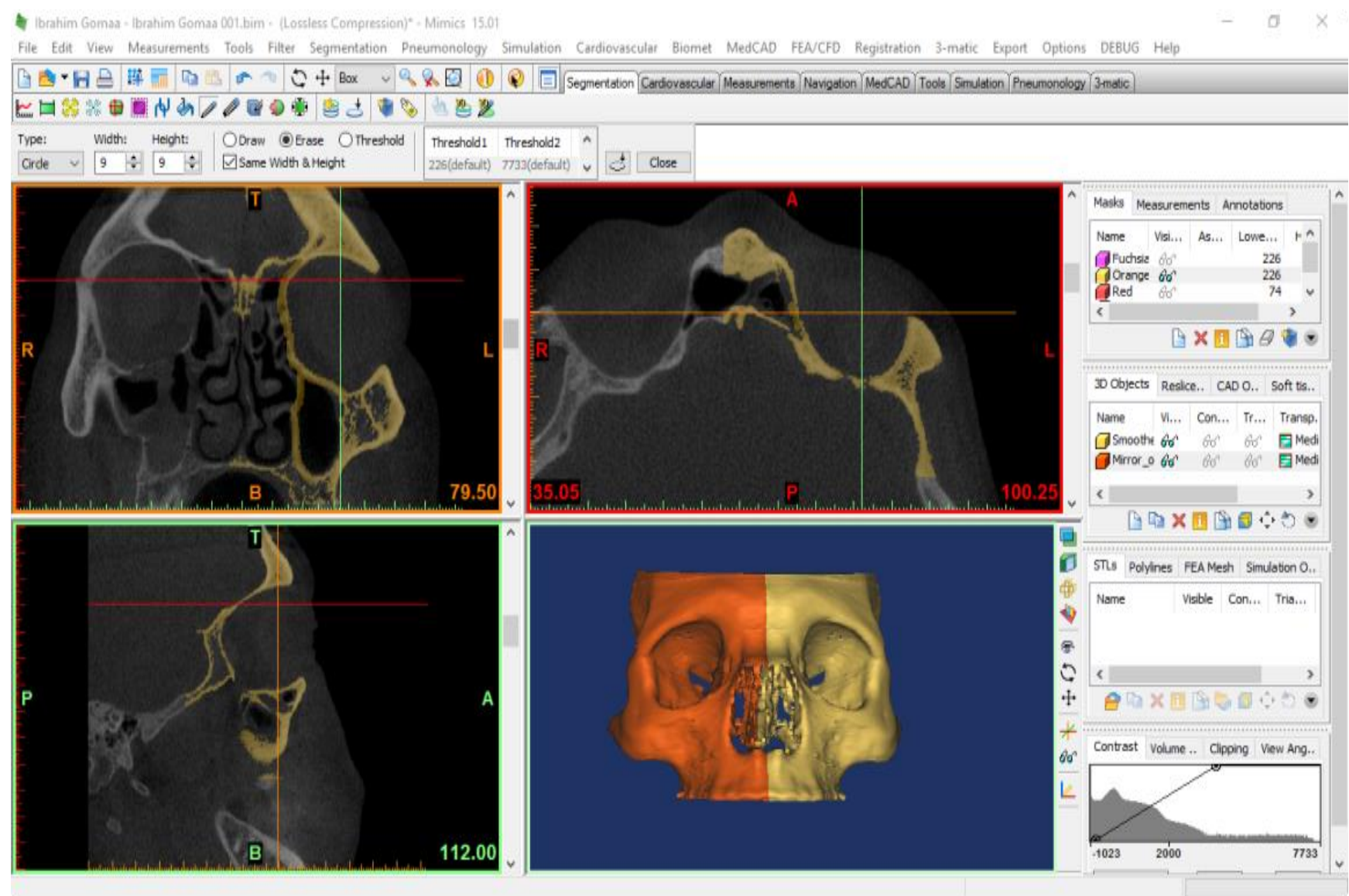

Figure (2): Mirror imaging technology using Mimics software overlapping the unaffected side onto the fractured one ad an STL model was designed.

\section{Operative technique:}

After general anesthesia was established, the anesthetic tube was secured to the patients head and an antiseptic eye ointment was applied carefully to the palpebral fissures. The surgical field was scrubbed with antiseptic solution and the patient was draped with sterile towels secured by towel clips leaving only the surgical field uncovered. A temporary lid suspension suture was carried out in order to stabilize and retract the lower lid. Subciliary incision was made through the skin only and hemostasis was achieved. Dissection through the orbicularis oculi muscle was done in a stepped myocutaneous flap design. The periosteum over the inferior orbital rim was sharply incised down to bone and elevated using a periosteal elevator to expose the fracture line. The globe was retracted carefully to explore the integrity of the orbital floor. Entrapped soft tissue was repositioned back into the orbit. An additional lateral eye brow incision was utilized to expose the frontozygomatic suture.

An intraoral maxillary vestibular incision was performed to expose midface fractures and to permit mobilization and reduction of the fractured zygomaticomaxillary complex. The fractured bones were freely mobilized and reduced into normal position. In group I, the preformed titanium mesh or/and plates were used as a template to assess the reduction of bones. Then, the reduced segments were fixed using the titanium plates and screws. (Figure 3) In group II, the titanium mesh or/and plates were manually bent to adapt to the surface of reduced bone fragments then fixed by the same way as in group I.

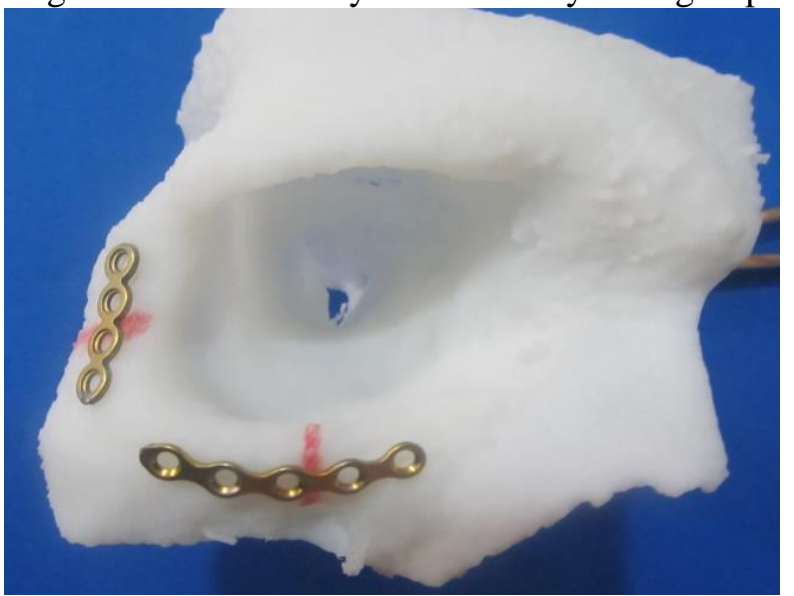

Figure (3): Preoperative contouring of the titanium miniplates on the 3D printed stereolithographic model. 
A drill pit rotating in a slow speed under copious saline irrigation was used to prepare bony osteotomies for the screws. The screws were inserted into bone through the plate holes and tightened carefully under saline irrigation with a suitable screwdriver. After implant insertion, it was imperative to perform a forced duction test in order to assure that the implant was not affecting the ocular motility.

The surgical field was thoroughly irrigated with saline to remove debris. The wound was then closed in layers. Resorbable Vicryle 3-0 suture material was used to close the muscle layer and subcutaneous tissue with adequate simple interrupted stitches. The skin was closed using 5-0 Prolene suture material. The intraoral incision was closed with Vicryle 3-0 suture material with locking continuous suturing technique. The suspensory lower lid suture was suspended to the patient forehead for two days after surgery and the eye was covered with a sterile eye patch.

The eye patch was maintained in place for 48 hours postoperatively. Local wound care was initiated as usual immediately after eye patch removal and maintained 3 times daily till patient discharge from admission. Patients were referred for postoperative ophthalmologic examination immediately after eye patch removal. Stitches were removed after one week.

\section{Postoperative assessment:}

\section{$>$ Clinical evaluation:}

Patients were evaluated clinically after one week, and at one, three and six months postoperatively for the following: wound site healing (infection and or dehiscence), diplopia gaze restriction, enophthalmos and exophthalmos.

\section{$>$ Radiographic evaluation:}

Postoperative CT scanning was performed immediately after surgery and 6 months postoperatively to evaluate:

1- The final outcome of the reconstructed orbit and position of the implant material.

2- To record the volumetric changes of the orbital cavities.

\section{VI: Statistical analysis of the data}

Data were fed to the computer and analyzed using IBM SPSS software package version 20.0.
(Armonk, NY: IBM Corp) Qualitative data were described using number and percent. The Kolmogorov-Smirnov test was used to verify the normality of distribution Quantitative data were described using range (minimum and maximum), mean, standard deviation and median. Significance of the obtained results was judged at the $5 \%$ level .

\section{RESULTS}

Ten male patients were included in the study after thorough clinical and radiographic examination confirming their eligibility criteria of selection. All of them have committed for every recall during the whole follow up observation period. They were divided randomly into two groups; five patients each. The patients ranged in age between $22.0-41.0$ years. The mean age of the patients of the group I was 30.80 \pm 7.36 years, while the mean age of the patients of the group II was $30.60 \pm 6.07$ years. Statistically no significant difference was found between the two groups. It was found that motor vehicle accident MVA account for $80.0 \%$ of the cases in both groups as a cause of trauma. Violence was responsible for $20.0 \%$ of the cases in both groups. No statistically significant difference was found between groups.

All cases of both groups showed an uneventful healing of their soft tissue and bony wounds without any infection, dehiscence nor plate exposure after completion of surgery. All orbits within both groups were favorably reduced and fixed. No cases of enophthalmos, exophthalmos, ectropion, entropion or visual acuity affection were recorded in both groups.

\section{Diplopia:}

In group I, 3 patients were presented with diplopia. After one week, one patient recovered from diplopia. The difference was statistically insignificant. At one, three and six months postoperatively, all patients have total recovery from diplopia. The difference was statistically significant. In group II, 3 patients were presented with diplopia. After one week, the 3 patients were still having diplopia. The difference was statistically insignificant. At one, 3 and six months postoperatively, all patients have total recovery from diplopia. The difference was statistically significant. Statistically non-significant difference was found between the two groups all over the observation periods. (Tables 1, 2) 
Table (1): Changes in diplopia along the different observation periods within groups

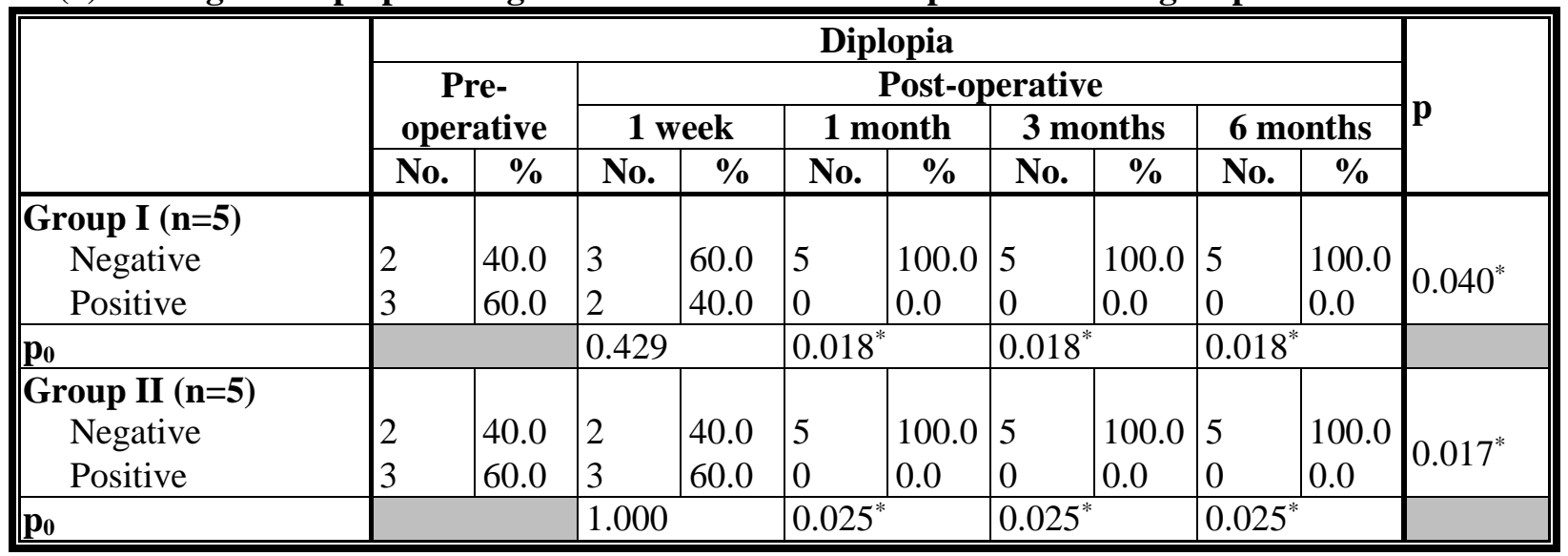

$p_{1}: p$ value for Cochran's test for comparing between the studied periods

$p_{0}$ : $p$ value for Post Hoc Test (Dunn's) for comparing between Pre-operative and each other periods *:

Statistically significant at $\mathrm{p} \leq \mathbf{0 . 0 5}$

Table (2): Comparison between the two studied groups according to diplopia

\begin{tabular}{|c|c|c|c|c|c|c|c|}
\hline \multirow{2}{*}{\multicolumn{2}{|c|}{ Diplopia }} & \multicolumn{2}{|c|}{$\begin{array}{l}\text { Group I } \\
(n=5)\end{array}$} & \multicolumn{2}{|c|}{$\begin{array}{l}\text { Group II } \\
(n=5)\end{array}$} & \multirow[t]{2}{*}{$\chi^{2}$} & \multirow[t]{2}{*}{${ }^{F E_{p}}$} \\
\hline & & No. & $\%$ & No. & $\%$ & & \\
\hline \multicolumn{2}{|l|}{\begin{tabular}{|c|}
$\begin{array}{c}\text { Pre-operative } \\
\text { Negative } \\
\text { Positive }\end{array}$ \\
\end{tabular}} & $\begin{array}{l}2 \\
3 \\
\end{array}$ & $\begin{array}{l}40.0 \\
60.0\end{array}$ & $\begin{array}{l}2 \\
3 \\
\end{array}$ & $\begin{array}{l}40.0 \\
60.0 \\
\end{array}$ & 0.000 & 1.000 \\
\hline \multirow{4}{*}{ 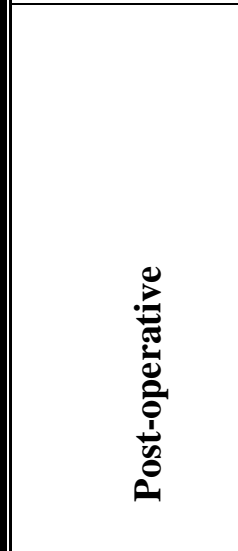 } & \begin{tabular}{|c|}
1 week \\
$\begin{array}{c}\text { Negative } \\
\text { Positive }\end{array}$
\end{tabular} & $\begin{array}{l}3 \\
2\end{array}$ & $\begin{array}{l}60.0 \\
40.0\end{array}$ & $\begin{array}{l}2 \\
3 \\
\end{array}$ & $\begin{array}{l}40.0 \\
60.0\end{array}$ & 0.400 & 1.000 \\
\hline & \begin{tabular}{|c|}
1 month \\
Negative \\
Positive \\
\end{tabular} & $\begin{array}{l}5 \\
0 \\
\end{array}$ & $\begin{array}{l}100.0 \\
0.0\end{array}$ & $\begin{array}{l}5 \\
0 \\
\end{array}$ & $\begin{array}{l}100.0 \\
0.0\end{array}$ & - & - \\
\hline & \begin{tabular}{|c|}
3 months \\
Negative \\
Positive \\
\end{tabular} & $\begin{array}{l}5 \\
0 \\
\end{array}$ & $\begin{array}{l}100.0 \\
0.0 \\
\end{array}$ & $\begin{array}{l}5 \\
0 \\
\end{array}$ & $\begin{array}{l}100.0 \\
0.0 \\
\end{array}$ & - & - \\
\hline & \begin{tabular}{|c|}
6 months \\
Negative \\
Positive \\
\end{tabular} & $\begin{array}{l}5 \\
0 \\
\end{array}$ & $\begin{array}{l}100.0 \\
0.0 \\
\end{array}$ & $\begin{array}{l}5 \\
0 \\
\end{array}$ & $\begin{array}{l}100.0 \\
0.0\end{array}$ & - & - \\
\hline
\end{tabular}

$\chi^{2}$ : Chi square test $\quad$ FE: Fisher Exact

p: $p$ value for comparison between the two studied groups

\section{Orbital volume:}

Descriptive statistics of orbital volume in the different groups are presented in (Table 3). The mean orbital volume of Group I (unaffected side) was $26.53 \pm 2.14 \mathrm{~cm}^{3}$ and the fractured side (immediately post-operative) was $26.55 \pm 2.15 \mathrm{~cm}^{3}$. While the mean orbital volume of Group II (unaffected side) was $25.06 \pm 1.56 \mathrm{~cm}^{3}$ and the fractured side (immediately post-operative) was $25.07 \pm 1.55 \mathrm{~cm}^{3}$. 
Table (3): Descriptive statistics of orbital volume in $\mathrm{cm}^{3}$ in each studied groups

\begin{tabular}{|c|c|c|c|c|c|c|c|c|}
\hline \multirow{2}{*}{ Groups } & \multirow{2}{*}{ Volume } & \multirow{2}{*}{ Min. } & \multirow{2}{*}{ Max. } & \multirow{2}{*}{ Mean } & \multirow{2}{*}{ \pm SD } & \multirow{2}{*}{ Median } & \multicolumn{2}{|c|}{$95 \% \mathrm{CI}$} \\
\hline & & & & & & & $\mathbf{L L}$ & $\mathbf{U L}$ \\
\hline \multirow{2}{*}{ Group I } & V0 & 23.62 & 29.07 & 26.53 & 2.14 & 26.16 & 23.88 & 29.19 \\
\hline & V2 & 23.63 & 29.07 & 26.55 & 2.15 & 26.17 & 23.88 & 29.22 \\
\hline \multirow{2}{*}{ Group II } & V0 & 23.55 & 27.39 & 25.06 & 1.56 & 24.51 & 23.13 & 26.99 \\
\hline & V2 & 23.58 & 27.41 & 25.07 & 1.55 & 24.51 & 23.14 & 27.0 \\
\hline
\end{tabular}

V0: orbital volume of the contralateral unaffected orbit.

V2: orbital volume of the ipsilateral traumatized orbit immediately after surgery.

Both study and control groups; showed a statistically insignificant difference in mean orbital volume in unaffected side and the affected side (immediately post-operative). There was a statistically insignificant difference between the two groups when comparing the mean orbital volume of the unaffected side and the immediately post-operative traumatized side. (Tables 4, 5)

Table (4): Comparison between orbital volume in $\mathrm{cm}^{3}$ of the traumatized orbit immediately after surgery

to the volume of the unaffected orbit in each group

\begin{tabular}{||l|l|l|l|l|l||}
\hline \multirow{5}{*}{} & \multicolumn{4}{|l|}{ Orbital volume $\mathbf{c m}^{\mathbf{3}}$} & \multirow{2}{*}{} \\
\cline { 2 - 6 } & V0 & V2 & $\mathbf{p}_{\mathbf{1}}$ \\
\cline { 2 - 7 } & Mean & $\mathbf{\pm S D}$ & Mean & $\mathbf{\pm S D}$ & \\
\hline Group I (n=5) & 26.53 & 2.14 & 26.55 & 2.15 & 0.259 \\
\hline Group II (n=5) & 25.06 & 1.56 & 25.07 & 1.55 & 0.288 \\
\hline
\end{tabular}

V0: orbital volume of the contralateral unaffected orbit.

V2: orbital volume of the ipsilateral traumatized orbit immediately after surgery.

$p_{1}$ : $p$ value for Paired t-test for comparing between V0 and V2

Table (5): Comparison between the two studied groups according to accuracy of reduction

\begin{tabular}{|c|c|c|c|c|c|c|}
\hline & \multicolumn{2}{|c|}{$\begin{array}{l}\text { Group I } \\
(n=5)\end{array}$} & \multicolumn{2}{|c|}{\begin{tabular}{|l}
$\begin{array}{l}\text { Group II } \\
(n=5)\end{array}$ \\
\end{tabular}} & \multirow[t]{2}{*}{$\mathbf{t}$} & \multirow[t]{2}{*}{$\mathbf{p}$} \\
\hline & Mean & \pm SD & Mean & \pm SD & & \\
\hline $\begin{array}{l}\text { Orbital volume } \mathbf{c m}^{\mathbf{3}} \\
\text { V0 } \\
\text { V2 }\end{array}$ & $\begin{array}{l}26.53 \\
26.55\end{array}$ & $\begin{array}{l}2.14 \\
2.15\end{array}$ & $\begin{array}{l}25.06 \\
25.07\end{array}$ & $\begin{array}{l}1.56 \\
1.55\end{array}$ & $\begin{array}{l}1.246 \\
1.250\end{array}$ & $\begin{array}{l}0.248 \\
0.247\end{array}$ \\
\hline
\end{tabular}

V0: orbital volume of the contralateral unaffected orbit.

V2: orbital volume of the ipsilateral traumatized orbit immediately after surgery.

t: Student t-test

p: $p$ value for comparison between the two studied groups

\section{DISCUSSION}

Ten patients with unilateral orbital fractures were involved in the study. They were randomly divided into two groups. Group I in which the used titanium plate / meshes were previously contoured by adapting them on an STL model 3D printed of the mirror imaged unaffected side of the patient. While in Group II, the patients were managed through the traditional reduction and fixation techniques and the titanium plates / mesh were adapted intraoperatively to the reduced bony fragments.
Only patients with unilateral orbital blow out fractures were included within this study. The contralateral unaffected orbit was used as a guide to create a mirror image overlay and fabricate an STL model preoperatively. Besides, the volume of this contralateral orbit was measured and considered as a reference point to compare the preoperative and postoperative traumatized orbit. Patients with a history of a traumatized / surgically operated orbit or optic nerve affection were excluded from the present study 
ejhm.journals.ekb.eg

as these situations will eventually affect the evaluation process and surgical outcomes.

In the present study diplopia was present in $60 \%$ of cases ( 3 patients from each group). After one week of surgical intervention, diplopia was present in $50 \%$ of cases (two patients within group I and three patients within group II). At one month postoperatively, the diplopia had resolved in all patients. This may be due to the early repair of the fracture. All cases were operated early within two weeks. It was found that there was no significant difference in postoperative diplopia when comparing patients treated with mirror image rapid prototype skull model (group I) and those treated without (group II). This is in disagreement with Randall et al. ${ }^{(13)}$ who found that the mirror image overlay group showed decreased postoperative diplopia in all fracture types and the need for revision surgery was greatly reduced.

Diplopia is a symptom that is frequently associated with orbital wall fractures. The thin floor of the orbit, typically medial to the infraorbital neuromuscular bundle, is broken and a piece of this bone is generally displaced downward into the maxillary sinus. Orbital tissue herniating into the sinus through the resulting defect in the orbital floor may become entrapped, causing diplopia ${ }^{(15)}$. Boyette et al. (16) found that the most common postoperative complication of orbital fracture is transient diplopia which will typically improve or resolve in a few weeks. However, the reported incidence of persistent diplopia ranges from $8 \%$ to $42 \%$. They concluded that the degree of occurrence strongly reinforces the need for intraoperative forced duction testing, as implant impingement can certainly be a cause for persistent diplopia. With good implant positioning, it is presumed that trauma to the muscle, fibrosis, or nerve paresis is the reason for diplopia.

In the absence of evident motor nerve laceration or extraocular muscle herniation or trapping into the fracture, recovery of diplopia in most adult cases is considered a matter of time ${ }^{(17,18)}$.

Hoşal et al. (19), Jamal et al. (20) and AlQurainy et al. ${ }^{(21)}$ came to an agreement that diplopia has improved few weeks to months following surgery and that surgical repair of blowout fractures within two weeks of trauma can decrease the incidence of residual diplopia. Ceylan et al. ${ }^{(22)}$ reported that there was no significant difference in postoperative primary gaze diplopia when comparing early versus delayed repair. Among twenty three patients with persistent diplopia, three $(7.6 \%)$ required strabismus surgery to correct persistent diplopia, although they had previously undergone orbital wall reconstruction; however, in four $(10.2 \%)$ patients, strabismus surgery was performed without fracture repair because of a history of trauma 28 months ago.

The most commonly fractured walls of the orbit are the floor and medial wall which mostly cause a significant orbital volume expansion. Combined fractures of the floor and medial wall are more likely than isolated orbital wall fractures to require treatment due to increased volume expansion. Orbital dystopia either vertical or axial may be the subsequent complication after the increase in orbital volume measurement which requires thorough management of the orbital fracture aiming at restoring the original shape and volume of the orbit, repositioning its contents and recovering ocular motility ${ }^{(23)}$.

To assess the accuracy of performed reduction, the volumetric measurement of the immediately reduced orbit was compared to the measurement of the contralateral unaffected side. The mean orbital volume of the traumatized orbit after reduction and fixation was $26.55 \pm 2.15 \mathrm{~cm}^{3}$ in group I and $25.07 \pm 1.55 \mathrm{~cm}^{3}$ in group II. Statistically, no significant difference in immediate postoperative orbital volumetric measurement when comparing patients treated with mirror image rapid prototype skull model (group I) with those repaired without (group II). This may be due to the proper and precise reduction and fixation of the orbital fracture. Six months after reduction and fixation of orbital fracture, no significant difference was found between both groups. This may be attributed to the maintained stability of the implant in situ.

This is in contrary to Tang et al. (24) who examined the application of individual digital design and rapid prototyping of specific titanium mesh implants in the reconstruction of orbital wall defects for different stages of orbital volume changes. They concluded that the degree of accuracy and rational of orbital volume reconstruction can be improved by appropriate individual digitalization design and rapid prototyping technology.

Ellis $\boldsymbol{e t} \boldsymbol{a l} .{ }^{(25)}$ have assessed the status of the internal orbit before and after reduction of ZMC fractures when treated without internal orbital reconstruction. They found that the size of the internal orbital defects increased slightly with ZMC reduction but the internal orbital fractures were realigned, and few had increases in orbital volume or soft tissue sagging into the sinuses decreasing its volumes. Examination of follow-up CT scans showed that the residual defects became smaller and that none of these patients had an increase in orbital volume or soft tissue sagging. 
ejhm.journals.ekb.eg

\section{CONCLUSIONS}

Based on the results of the study, it could be concluded that:

- The use of mirror image rapid prototype skull model for treatment of orbital fractures has no advantage over the traditional method.

- Though it is so costly, the use of advanced technology is fascinating and has a tremendous improvement in the diagnosis and treatment of orbital fractures and has a remarkable role in facilitating the precise contouring and adaptation of the titanium plates / mesh needed for fracture repair.

\section{REFERENCES}

1. Jaquiery C, Aeppli C, Cornelius $P$ et al. (2007): Reconstruction of orbital wall defects: critical review of 72 patients. Int J Oral Maxillofac Surg., 36: 193-9.

2. Hwang K, You S (2010): Analysis of facial bone fractures: An 11-year study of 2,094 patients. Ind J Plast Surg., 43:42-8.

3. Erdmann D, Follmar K, Debruijn M et al. (2008): A retrospective analysis of facial fracture etiologies. Ann Plast Surg., 60: 398-403.

4. Magarakis M, Mundinger G, Kelamis J et al. (2012): Ocular injury, visual impairment, and blindness associated with facial fractures: a systematic literature review. Plast Reconstr Surg., 129: 227-33.

5. Rinna C, Ungari C, Saltarel A et al. (2005): Orbital floor restoration. J Craniofac Surg., 16: 968-72.

6. Dobrovăț B, Popescu R, Nemtoi A et al. (2011): Orbital trauma: from anatomy to imaging patterns - a pictorial review. Romanian Neurosurgery, 4: 525-32.

7. Toro C, Robiony M, Costa F (2007): Feasibility of preoperative planning using anatomical facsimile models for mandibular reconstruction. Head Face Med., 3: 5-14.

8. Exadaktylos A, Sclabas G, Smolka K (2005): The value of computed tomographic scanning in the diagnosis and management of orbital fractures associated with head trauma: a prospective, consecutive study at a level I trauma center. J Trauma, 58: 336-41.

9. Bibb R, Eggbeer D, Evans $P$ (2009): Rapid manufacture of custom fitting surgical guides. Rapid Prototyping J., 15: 346-53.

10. Levine J, Patel A, Saadeh P et al. (2012): Computeraided design and manufacturing in craniomaxillofacial surgery: the new state of the art. J Craniofac Surg., 23: 288-93.
11. Mehra P, Miner J, Innocenzo R et al. (2011): Use of 3-D Stereolithographic Models in Oral and Maxillofacial Surgery. J Maxillofac Oral Surg., 10: 613.

12. Liu P, Wong T, Fang J et al. (2014): 3D stereolithographic modeling technique for hemimandibular reconstruction, report of a case with innovation technique. Open J Dent Oral Med., 2: 9-13.

13. Randall A, Chang S, Cudejkova $M$ et al. (2013): Computer-guided orbital reconstruction to improve outcomes. JAMA Facial Plast Surg., 15: 113-20.

14. Park S, Choi J, Koh K et al. (2015): Mirror imaged rapid prototype skull model and pre molded synthetic scaffold to achieve optimal orbital cavity reconstruction. J Oral Maxillofac Surg., 73: 1540-53.

15. Yoo Y, Yang H, Kim N et al. (2017): Pediatric orbital wall fractures: Prognostic factors of diplopia and ocular motility limitation. PLoS One, 12: 1-12.

16. Boyette J, Pemberton J, Bonilla-Velez J (2015): Management of orbital fractures: challenges and solutions. Clin Ophthalmol., 9: 2127-37.

17. Su Y, Shen Q, Lin M et al. (2015): Diplopia of pediatric orbital blowout fractures: A retrospective study of 83 patients classified by age groups. Medicine, 94: 1-6.

18. Cope M, Moos K, Speculand B (1999): Does diplopia persist after blow-out fractures of the orbital floor in children? Br J Oral Maxillofac Surg., 37: 46-51.

19. Hoşal B, Beatty R (2002): Diplopia and enophthalmos after surgical repair of blowout fracture. Orbit., 21: 2733.

20. Jamal B, Pfahler S, Lane K et al. (2009): Ophthalmic injuries in patients with zygomaticomaxillary complex fractures requiring surgical repair. J Oral Maxillofac Surg., 67: 986-9.

21. Al-Qurainy I, Dutton G, Stassen L et al. (1991): Diplopia following midfacial fractures. $\mathrm{Br} \mathrm{J}$ Oral Maxillofac Surg., 29: 302-7.

22. Ceylan O, Uysal Y, Mutlu F et al. (2011): Management of diplopia in patients with blowout fractures. Ind J Ophthalmol., 5: 461-4.

23. Chi R, Davies B (2013): Combined orbital floor and medial wall fractures involving the inferomedial strut: repair technique and case series using preshaped porous polyethylene/titanium implants. Craniomaxillofac Trauma Reconstr., 6: 161-70.

24. Tang W, Guo L, Long $J$ et al. (2010): Individual design and rapid prototyping in reconstruction of orbital wall defects. J Oral Maxillofac Surg., 68: 562-70.

25. Ellis E, Reddy L (2004): Status of the internal orbit after reduction of zygomaticomaxillary complex fractures. J Oral Maxillofac Surg. , 62: 275-83. 